\title{
Entrance channel effect in the incomplete fusion reactions
}

\author{
Abhishek Yadav ${ }^{1, a}$, Vijay R. Sharma ${ }^{1}$, Pushpendra P. Singh ${ }^{2}$, Manoj K. Sharma ${ }^{3}$, Devendra P. Singh ${ }^{1}$, Unnati ${ }^{1}$, R. Kumar ${ }^{4}$, \\ B. P. Singh ${ }^{1}$, R. Prasad ${ }^{1}$ and R. K. Bhowmik ${ }^{4}$ \\ ${ }^{1}$ Department of Physics, A. M. University, Aligarh (U.P.)-202 002, India \\ ${ }^{2}$ INFN-Laboratori Nazionali di Legnaro, I-35020 Legnaro, Italy \\ ${ }^{3}$ Physics Department, S.V.College, Aligarh (U.P.), India \\ ${ }^{4}$ NP-Group, Inter University Accelerator Centre, New Delhi-110067, India
}

\begin{abstract}
In the present work the effect of various entrance channel parameters on incomplete fusion strength and the reaction dynamics in ${ }^{12} \mathrm{C}+{ }^{159} \mathrm{~Tb}$ system at energies $\approx 4-7 \mathrm{MeV} / \mathrm{A}$ have been investigated by measuring the excitation functions of individual reaction channels. Experimental excitation functions have been analyzed in the framework of compound nucleus decay using statistical model code PACE4. Analysis of data suggests the production of $x \mathrm{n} / \mathrm{p} x \mathrm{n}$-channels via complete fusion of ${ }^{12} \mathrm{C}$ with ${ }^{159} \mathrm{~Tb}$, as these are found to be well reproduced by PACE4 predictions, while, a significant enhancement in the excitation functions of $\alpha$-emitting channels has been observed over the theoretical ones. This enhancement has been attributed due to incomplete fusion. For better insight into the underlying dynamics, fraction of incomplete fusion to the total fusion has been deduced and compared with ${ }^{16} \mathrm{O}+{ }^{159} \mathrm{~Tb}$ and other nearby systems as a function of various entrance channel parameters. The fraction of incomplete fusion has been found to be sensitive to the projectile type, energy and entrance-channel mass-asymmetry.
\end{abstract}

\section{Introduction}

Recently, the unexpected presence of incomplete fusion (ICF) at low projectile energies (i.e. $\approx 4-7 \mathrm{MeV} / \mathrm{A}$ ), where only complete fusion $(\mathrm{CF})$ is expected to be the sole contributor to the total fusion cross-section has attracted both experimentalist as well as theorists [1-9]. In recent studies [5-10] a substantial fraction of ICF has been observed at slightly above barrier energies. The concept of incomplete mass transfer in HI-reactions has originated after the pioneering experimental observation of "fast- $\alpha$-particles" by Britt and Quinton [11] at relatively higher energies $\approx 10.5 \mathrm{MeV} / \mathrm{A}$. Since then, several theoretical models have been proposed to understand the ICF-reaction dynamics [12-16], however, these models explain the experimental data up to some extent only, that too at energies $\geq 10 \mathrm{MeV} / \mathrm{A}$ or so. Further, the study of ICF reactions got resurgent interest after the observation of large ICF contribution at energies $\approx 4-7 \mathrm{MeV} / \mathrm{A}[5-10]$. In one of our recent letters [7], it has been observed that the ICF component at fixed incident energy is associated to increased mean input angular momenta ( $\ell$-values) for successively opened $\alpha$ emitting channels. As per the generalized concept of input angular momenta, all $\ell$-values from $\ell=0$ to $\ell_{\text {crit }}$ are associated with central and/or near central interactions, where projectile-target nuclei amalgamate and thus, form a completely fused composite system, which may eventually decay via usual modes [15]. However, for the higher $\ell$-values ICF sets in, where, the sum of repulsive Coulomb and centrifugal potential overcomes the attractive nuclear potential. As a result, pocket in the effective potential disappears and fusion of interacting nuclei is hindered due to the higher input angular momenta ( $\ell$-values) than the fusion limit $\left(\ell_{\text {crit }}\right)$. To release excess input angular momenta imparted into the system the projectile breaks up into its constituents. It may be pointed out that the projectile break up modifies the picture of fusion of two strongly bound nuclei as; (i) complete fusion (CF), where projectile and/or its all fragments may fuse with the target nucleus after projectile breakup and (ii) incomplete fusion (ICF), defined as the fusion of only one of the fragments with target nucleus and remnant moving in forward cone with projectile velocity and (iii) no capture breakup (NCBU), where no breakup fragment is captured by the target nucleus. As such, the total fusion cross-section (TF here after) may be defined as the sum of CF and ICF crosssections. Earlier experiments [5-8] have conclusively demonstrated the presence of substantial ICF contribution even at low energies but limited only to a few projectiletarget combinations. Hence, in order to investigate the role of various entrance channel parameters, e.g., (i) projectile type/energy, and (ii) mass-asymmetry $\left(\mu_{\mathrm{A}}=\mathrm{A}_{\mathrm{T}}\right.$

\footnotetext{
a abhishekyadav117@gmail.com
}

This is an Open Access article distributed under the terms of the Creative Commons Attribution-Noncommercial License 3.0, which permits unrestricted use, distribution, and reproduction in any noncommercial medium, provided the original work is properly cited. 
$\left./ \mathrm{A}_{\mathrm{T}}+\mathrm{A}_{\mathrm{P}}\right)$ on the onset and fraction of ICF, the excitation functions (EFs) for residues populated in the ${ }^{12} \mathrm{C}+{ }^{159} \mathrm{~Tb}$ interactions at energies $\approx 4-7 \mathrm{MeV} / \mathrm{A}$ have been measured and obtained results have been compared with other nearby systems to obtain some systematics. Experimental data is analysed within the framework of theoretical model code PACE4 based on equilibrated compound nucleus decay. In one of our earlier experiments the excitation functions (EFs) and forward recoil ranges for ${ }^{16} \mathrm{O}+{ }^{159} \mathrm{~Tb}$ system have been measured at $\approx 5-7 \mathrm{MeV} / \mathrm{A}$ [5], where substantial contribution of ICF reaction dynamics have been found. The present projectile-target combination $\left({ }^{12} \mathrm{C}+{ }^{159} \mathrm{~Tb}\right)$ has been chosen to draw some conclusions on entrance channel effect and on the onset and strength of ICF by comparing it with a few nearby systems.

\section{Experiment and Data Reduction}

The experiments have been performed at the Inter University Accelerator Center (IUAC), New Delhi, India, using activation technique followed by off-line $\gamma$-ray spectroscopy [5-8]. The irradiation of the samples have been carried out in the General Purpose Scattering Chamber having an in-vacuum transfer facility, which has been used to minimize the lapse time between the stop of the irradiation and beginning of the counting of the activity induced in the target-catcher assembly. The isotopically pure, self-supporting ${ }^{159} \mathrm{~Tb}$ targets of thickness $\approx 1.2-2.5 \mathrm{mg} / \mathrm{cm}^{2}$ were prepared by rolling technique. The Al-foils were also prepared by the rolling technique, which served as energy degrader as well as catcher foils $\left(\approx 1.5-2.5 \mathrm{mg} / \mathrm{cm}^{2}\right)$ to trap the recoiling residues produced during the interaction of ${ }^{12} \mathrm{C}+{ }^{159} \mathrm{~Tb}$. In order to cover a wide energy region in a single irradiation, energy degradation technique has been used. In the present experiment five stacks, each having three target-catcher foil assemblies were irradiated at energies $\approx 59,70,73,85$ and $88 \mathrm{MeV}$. Keeping the half-lives of interest in mind, irradiations were carried out for $\approx 8-10 \mathrm{~h}$ duration for each stack. The Pelletron crew provided a nearly constant beam current $\approx 30 \mathrm{nA}$ throughout the irradiations. A Faraday cup, which was placed behind the target-catcher foil assembly, was used to measure the integrated beam current, for every $120 \mathrm{~s}$, so as to correct for the variation in the beam intensity during the irradiation, which is particularly significant for shortlived radio-nuclides. The activities produced in each target-catcher foil assembly have been recorded using a high resolution pre-calibrated HPGe detector coupled to a PC through CAMAC based data acquition software CANDLE [17]. A $50 \mathrm{~Hz}$ pulser was used to determine the dead time. The detector-sample separation was adjusted to keep the dead-time below $10 \%$ during the counting so as to minimize the pile up effects. The efficiency calibration of the detector in the specified geometry was carried out using a standard ${ }^{152} \mathrm{Eu}$ source of known strength at various source-detector separations. A typical $\gamma$-ray spectrum for the ${ }^{12} \mathrm{C}+{ }^{159} \mathrm{~Tb}$ interaction at $\approx 87.31 \pm$ $0.69 \mathrm{MeV}$ is shown in the Fig.1, where some of the prominent $\gamma$-lines have assigned to different reaction products, populated via $\mathrm{CF}$ and/or ICF channels.

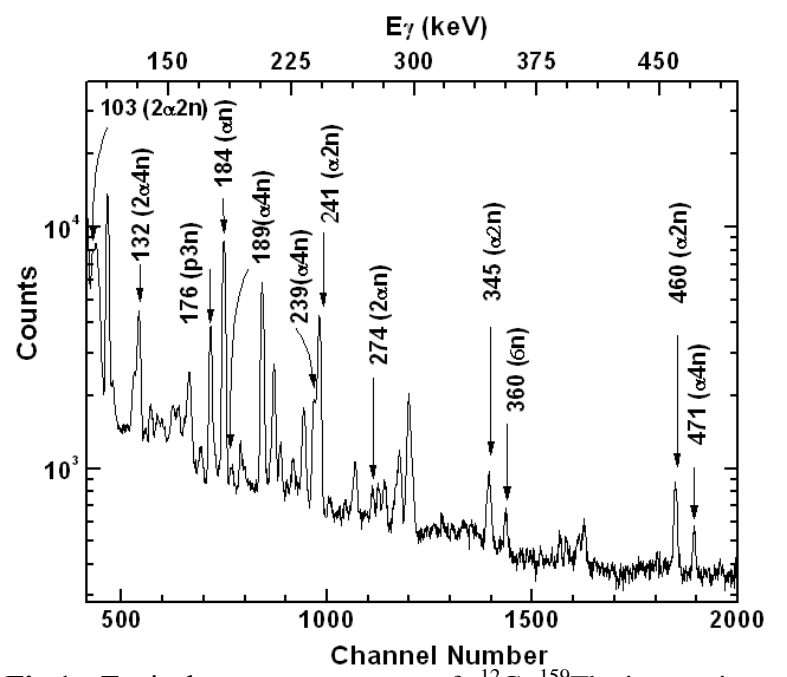

Fig.1. Typical $\gamma$-ray spectrum of ${ }^{12} \mathrm{C}+{ }^{159} \mathrm{~Tb}$ interactions at $\approx 87.31 \mathrm{MeV}$, indicating different $\mathrm{CF}$ and/or ICF residue $\gamma$-lines.

Further, confirmation of the identified reaction products has been made by the decay curve analysis. Nuclear data on radio-nuclides, such as the corresponding $\gamma$-ray abundances and half lives were taken from ref [18]. The production cross sections of the reaction products have been determined using the standard formulation (for details see ref. 7-8). In the present work the EFs for ${ }^{168,168 \mathrm{~m}} \mathrm{Lu}(3 \mathrm{n}),{ }^{167} \mathrm{Lu}(4 \mathrm{n}),{ }^{165} \mathrm{Lu}(6 \mathrm{n}),{ }^{167} \mathrm{Yb}(\mathrm{p} 3 \mathrm{n}),{ }^{165} \mathrm{Tm}$ $(\alpha 2 n),{ }^{163} \mathrm{Tm}(\alpha 4 n),{ }^{161} \mathrm{Ho}(2 \alpha 2 n),{ }^{160} \mathrm{Ho}(2 \alpha 3 \mathrm{n})$ and ${ }^{159} \mathrm{Ho}$ $(2 \alpha 4 n)$ residues have been measured. The errors in the measured production cross-sections of different radionuclides may arise due to various factors and the overall errors including statistical errors are estimated to be $\leq 15 \%$, excluding the uncertainty in branching ratio, decay constant, etc.

\section{Interpretation of excitation functions}

Information regarding the reaction mechanism in the ${ }^{12} \mathrm{C}+{ }^{159} \mathrm{~Tb}$ interactions may be obtained by comparing the experimentally measured EFs with the theoretical ones calculated using code PACE4 [19]. The code PACE4 is based on Hauser-Feshbach theory for CN-decay and uses statistical approach of $\mathrm{CN}$ de-excitation by Monte Carlo procedure. The code uses the BASS model [20] for CF cross section calculation. The default optical model parameters for neutrons, protons and $\alpha$-particles are used. This code has been modified to take into account the excitation energy dependence of the level density parameter using the prescription of Kataria et al. [21]. It should be pointed out that the ICF and PE-emission are not taken into consideration in this code.

\subsection{The xn \& pxn-channels: mainly populated via CF}

From the analysis of experimental data, activities corresponding to nuclide ${ }^{168,168 \mathrm{~m}} \mathrm{Lu}\left(\mathrm{T}_{1 / 2}=6.7 \mathrm{~min} \& 5.5\right.$ $\min ),{ }^{167} \mathrm{Lu}\left(\mathrm{T}_{1 / 2}=51.5 \mathrm{~min}\right),{ }^{165} \mathrm{Lu}\left(\mathrm{T}_{1 / 2}=10.74 \mathrm{~min}\right)$ and ${ }^{167} \mathrm{Yb}\left(\mathrm{T}_{1 / 2}=17.5 \mathrm{~min}\right)$, which are populated via $3 \mathrm{n}, 4 \mathrm{n}$, 
6n and p3n-channels, respectively, have been determined. It may be pointed out that the residue corresponding to $5 n$-channel could not be measured due to its short halflife. However, p2n-channel produces stable residue. The experimentally measured and theoretically calculated EFs for residues populated via $x \mathrm{n} \&$ p $x \mathrm{n}$-channels are shown in Fig.2. In the present work the theoretical calculations have been done with code PACE4. The level density parameter ' $\mathrm{a}$ ' $(=\mathrm{A} / \mathrm{K})$, is one of the important parameters, where, $\mathrm{A}$ is the mass number of the nucleus and $\mathrm{K}$ is a free parameter. The value of $\mathrm{K}$ may be varied to match the experimental data. In the present work as well as in our previous studies, the measured EFs have been reproduced satisfactorily using ' $\mathrm{a}$ ' $=\mathrm{A} / 8 \mathrm{MeV}^{-1}$ for this mass-region. Since, the theoretical calculation of PACE4 for the residues populated via $x \mathrm{n}$-channels $(x=3,4,6)$ and the production cross-section for ${ }^{167} \mathrm{Yb}(\mathrm{p} 3 \mathrm{n})$ residue deduced after the subtraction of precursor contribution [22] satisfactorily reproduce the experimentally measured excitation functions, it may be inferred that these channels are populated via CF only (see Fig.2.). In this figure the $\Sigma \sigma_{\mathrm{CF}}$ has been corrected for the contribution of $5 \mathrm{n} \& \mathrm{p} 2 \mathrm{n}$-channels obtained from PACE4 predictions.

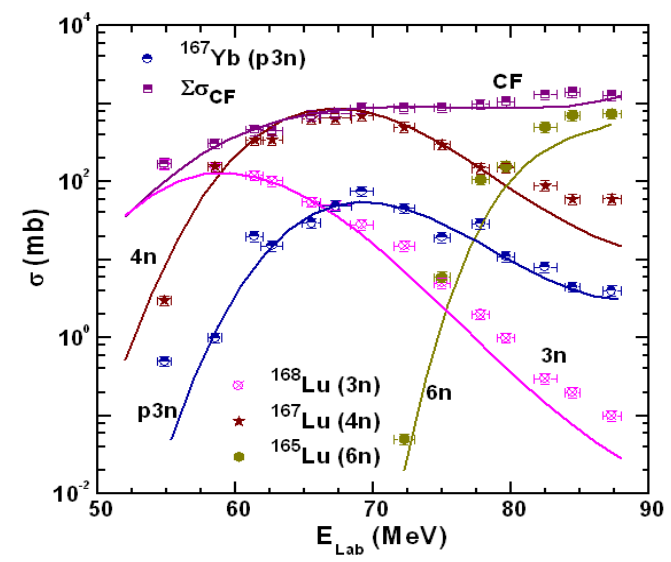

Fig.2. The experimentally measured and theoretically calculated EFs for $x \mathrm{n} \& \mathrm{p} x \mathrm{n}$-channels populated in ${ }^{12} \mathrm{C}+{ }^{159} \mathrm{~Tb}$ interactions. The solid lines are the theoretical predictions of code PACE4, done using suitable set of parameters (see text for details).

\section{$3.2 \alpha$-emitting channels: enhancement over PACE4 predictions}

As the EFs for $x \mathrm{n} \&$ pxn-channels are satisfactorily reproduced via PACE4 calculations and hence, it is reasonable to assume that the parameters used in these calculations are suitable for the present system. Therefore, the same set of input parameters has also been used to fit the EFs of $\alpha$-emitting channels. Note that in the case of $\alpha$-emitting-channels, the residue may be formed via two ways: (i) by $\mathrm{CF}$ of ${ }^{12} \mathrm{C}$ followed by the formation of an excited $\mathrm{CN}$ from which evaporation of neutrons and $\alpha$-particle may take place, or (ii) the partial fusion of ${ }^{12} \mathrm{C}$ ion when it breaks into ${ }^{8} \mathrm{Be}$ and $\alpha$, where ${ }^{8} \mathrm{Be}$ fuses with the target nucleus leaving the $\alpha$-particle as spectator and vice-versa. The measured cross-sections corresponding to $\alpha x \mathrm{n}\left({ }^{167-x} \operatorname{Tm}(x=2,4)\right)$ and $2 \alpha x n-$ channels $\left({ }^{163-x}\right.$ Ho $\left.(x=2,3,4)\right)$ are shown in Fig.3 (a-b). In this Fig.3 (a-b) the black line are the theoretical calculations done with the parameters as used to reproduce the $x \mathrm{n} / \mathrm{p} x \mathrm{n}$-channels. Again, the residue ${ }^{164} \mathrm{Tm}$ $(\alpha 3 n)$ could not be measured experimentally due to its short half-life. It is very much evident from the Fig.3 (ab) that there is a significant enhancement in experimental cross-section $\Sigma \sigma_{\text {zoxn }}\left(\right.$ expt) over theoretical $\Sigma \sigma_{\text {zoxn }}($ PACE4) for both $\alpha x \mathrm{n}$ and $2 \alpha x \mathrm{n}$-channels. Hence, it may be concluded that all the $\alpha$-emitting channels $(\alpha x n \& 2 \alpha x n)$ have significant contributions from ICF reaction processes.
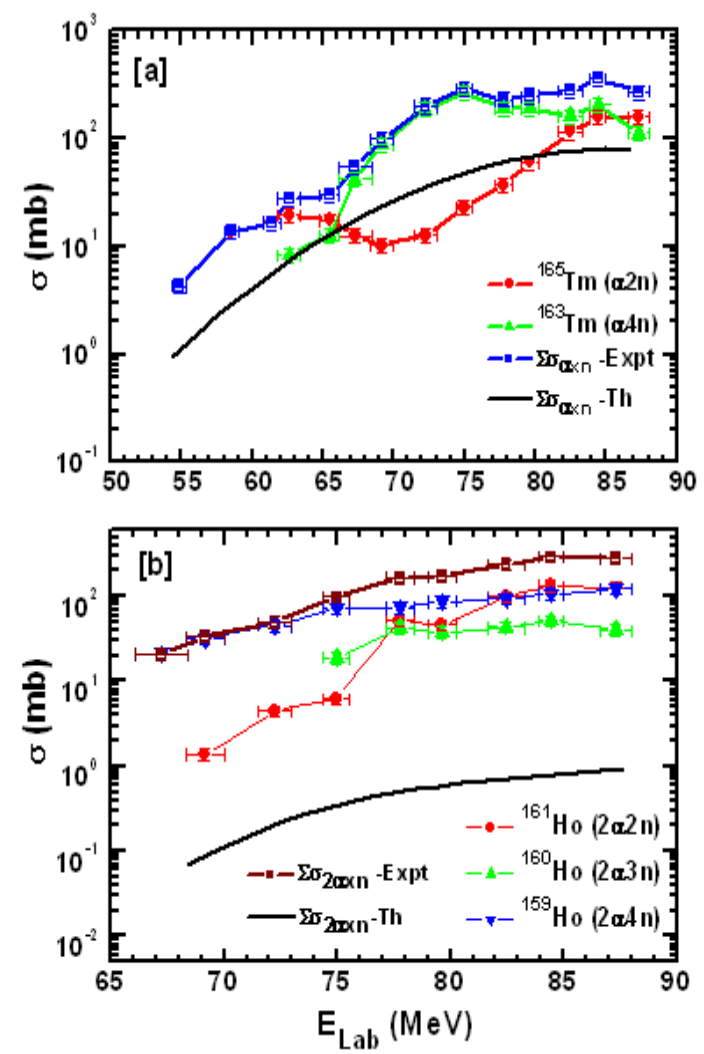

Fig.3. The experimentally measured excitation functions for $\alpha$ emitting channels. The PACE4 calculations (solid black line) have been done with the same set of parameters as used to reproduce $x \mathrm{n} \& \mathrm{p} x \mathrm{n}$-channels. The solid lines through data points are just to guide the eye (see text for details).

\subsection{Incomplete fusion fraction}

Since, it is evident from analysis of EFs that ICFreactions contribute significantly to the evaporation residue cross-sections. As such, an attempt has been made to deduce the ICF contribution from experimentally measured EFs, using the prescription of Gomes et al., [3]. The ICF-contribution for individual channels has been deduced by subtracting CF cross-sections $\Sigma \sigma_{\mathrm{CF}}$ (predicted by code) from the experimentally measured cross sections $\sigma_{\mathrm{TF}}$ at each studied energy. The ICF contributions so deduced for all measured ICF-channels $\left(\Sigma \sigma_{\text {ICF }}\right)$ and the sum of cross-sections for all CF-channels $\left(\Sigma \sigma_{\mathrm{CF}}\right)$ obtained from theoretical model predictions are plotted along with the total fusion cross-section $\left(\sigma_{\mathrm{TF}}=\Sigma \sigma_{\mathrm{CF}}+\Sigma \sigma_{\mathrm{ICF}}\right)$ in Fig. 4(a). The projectile energy normalized with Coulomb barrier $\left(E_{\text {Proj }} / V_{b}\right)$ in Fig.4 (a), has been chosen to compare different projectile-target combinations in a plot, as 
reported in ref. [3]. The lines drawn in these figures are just to guide the eyes. It is not out of place to mention that the $\sigma_{\mathrm{TF}}$ has been corrected for the missing $5 \mathrm{n}, \mathrm{p} 2 \mathrm{n}$ and $\alpha 3 n$-channels (which could not be measured experimentally) by their PACE4 values. As such, in the quoted $\Sigma \sigma_{\text {ICF }}$ values shown in Fig.4 (a), the ICF contribution of $\alpha 3 \mathrm{n}$-channel could not be included. Hence, the $\Sigma \sigma_{\text {ICF }}$ may be taken at least as the lower limit of ICF contribution.
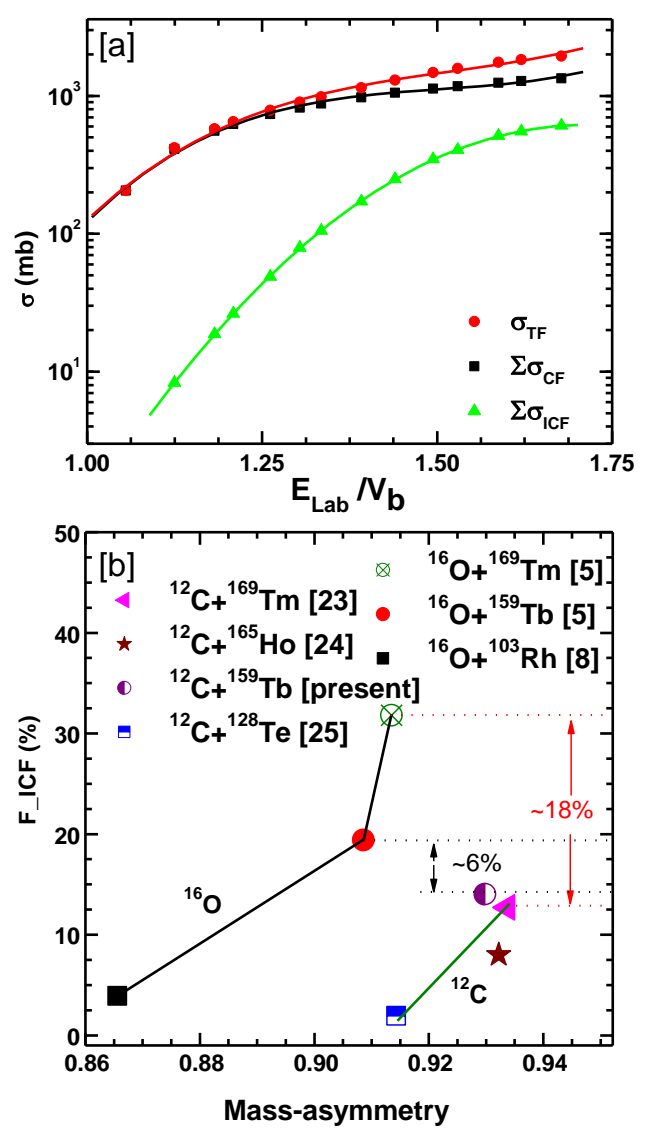

Fig.4. The TF with $\mathrm{CF} \& \mathrm{ICF}$ cross-sections as a function of normalized projectile energy (upper panel); however, the lower panel contains the comparison of near-by system at same relative velocity (details see in text).

\section{Remarks on the effect of projectile type}

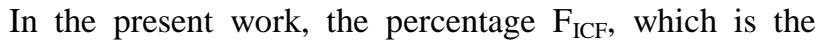
measure of relative strength of ICF contribution to total fusion, may be defined as, $\mathrm{F}_{\mathrm{ICF}}(\%)=\left(\Sigma \sigma_{\mathrm{ICF}} / \sigma_{\mathrm{TF}}\right) \times 100$ have been deduced. In order to see the effect of the type of projectile, in Fig.4 (b) the $\mathrm{F}_{\mathrm{ICF}}$ have been plotted as a function of mass-asymmetry of the interacting partners for different projectile-target combinations at a constant relative velocity $\left(v_{\text {rel }}=0.053\right)$. It is clear from this figure, that for the same target ${ }^{169} \mathrm{Tm}$ the $\mathrm{F}_{\mathrm{ICF}}$ is $\approx 18 \%$ larger for ${ }^{16} \mathrm{O}$ than for ${ }^{12} \mathrm{C}$ as projectile. Similarly, for ${ }^{159} \mathrm{~Tb}$ target the $\mathrm{F}_{\mathrm{ICF}}$ is $\approx 6 \%$ more for ${ }^{16} \mathrm{O}$ beam as compared to ${ }^{12} \mathrm{C}$. Moreover, it may be observed from this figure that $\mathrm{F}_{\mathrm{ICF}}$ increases with mass-asymmetry, individually for each ${ }^{16} \mathrm{O}$ and ${ }^{12} \mathrm{C}$ projectiles. It may also be observed that for both ${ }^{169} \mathrm{Tm}$ and ${ }^{159} \mathrm{~Tb}$ target nuclides, the ICF-contribution for
${ }^{16} \mathrm{O}$ projectile is larger than the ICF-contribution for ${ }^{12} \mathrm{C}$ projectile. Besides the excess of alpha cluster in ${ }^{16} \mathrm{O}$, one of the possible reasons for higher $\mathrm{F}_{\text {ICF }}$ in case of ${ }^{16} \mathrm{O}$ may be the fact that it has relatively less $\mathrm{Q}_{\alpha}(-7.16 \mathrm{MeV})$ as compared to ${ }^{12} \mathrm{C}, \mathrm{Q}_{\alpha}(-7.37 \mathrm{MeV})$ value. From Fig.4 (b) it may be concluded that $\mathrm{F}_{\mathrm{ICF}}$ depends not only on the massasymmetry of the interacting partners but also on the type of projectile, which plays an important role in the underlying reaction dynamics.

\section{Acknowledgements}

The authors thank to the Director, IUAC, New Delhi, India, for providing all the necessary facilities to carry out this work. AY thanks to the UGC for providing financial support in the form of SRF. BPS, MKS and RP thank to DST and UGC for providing financial support.

\section{References}

1. A. Diaz-Torres and I. J. Thompson, Phys. Rev. C 65, 024606 (2002), Phys. Rev. Lett. 98, 152701 (2007).

2. D. J. Parker et al., Phys. Rev. C 39, 2256 (1989).

3. P. R. S. Gomes, el al., Phys. Rev. C 73, 064606 (2006), Phys. Lett. B 601, 20 (2004).

4. M. Dasgupta, et al., Phys. Rev. Lett. 82, 1395 (1999), Nucl. Phys. A 787, 144-149 (2007).

5. Pushpendra P. Singh, et al., Phys. Rev. C 77, 014607 (2008); Euro. Phys. J. A 34, 29-39 (2007).

6. Pushpendra P. Singh, et al., Phys. Rev. C 78, 017602 (2009), and references therein.

7. Pushpendra P. Singh, et al., Phys. Lett. B 671, 20-24 (2009), Phys. Rev. C 80, 064603 (2009) and references therein.

8. Unnati Gupta, et al., Nucl. Phys. A 811, 77-92 (2008), Phys. Rev. C80, 024613 (2009).

9. L. F. Canto, et al., Phys. Rev. C 58, 1107 (1998).

10. M. Dasgupta, el al., Phys. Rev. C 70, 024606 (2004).

11. H. C. Britt and A. R. Quinton, Phys. Rev. 124, 877 (1961).

12. T. Inamura, et al., Phys. Lett. 68B, 51 (1977), Phys. Lett. 84B, 71 (1982); Phys. Rev. C32, 1539 (1985).

13. T. Udagawa and T. Tamura, Phys. Rev. Lett. 45, 1311 (1980).

14. J. R. Wu, and I. Y. Lee, Phys. Rev. Lett. 45, 8 (1980)

15. J. Wilczynski, et al., Phys. Rev. Lett. 45, 606 (1980); Nucl. Phys. A 373, 109 (1982).

16. I. M. Brancus, et al., Phys. Rev. C 42, 2157 (1990).

17. B. P. Ajith Kumar et. al., DAE SNP, 2001, Kolkotta.

18. E. Browne, R.B. Firestone, Table of Radioactive Isotopes, Wiley, New York, 1986.

19. A. Gavron, Phys. Rev.C 21, 230 (1980).

20. R. Bass, Nucl. Phys. A 231, 45-63 (1974).

21. S. K. Kataria et al., Phys. Rev. C 18, 549 (1978).

22. M. Cavinato et al., Phys. Rev. C 52, 2577 (1995).

23. K. Sudarshan et al., Phys. Rev. C 69, 027603 (2004).

24. S. Gupta et al., Phys. Rev. C 61, 064613 (2000).

25. M. K. Sharma et al., Joul. of Phys. Society of Japan 72, 1917 (2003). 